\title{
Communication-type smartphone application can contribute to reducing elapsed time to reperfusion therapy
}

\author{
Kenichiro Sakai ${ }^{1}$ D $\cdot$ Takeo Sato $^{1} \cdot$ Teppei Komatsu $^{1} \cdot$ Hidetaka Mitsumura $^{1} \cdot$ Yasuyuki Iguchi $^{1} \cdot$ Toshihiro Ishibashi $^{2} \cdot$ \\ Yuichi Murayama ${ }^{2} \cdot$ Kohei Takeshita $^{3} \cdot$ Hiroyuki Takao $^{2,3}$
}

Received: 6 January 2021 / Accepted: 20 February 2021 / Published online: 26 February 2021

(C) Fondazione Società Italiana di Neurologia 2021

\begin{abstract}
Background and objectives Communication-type medical smartphone applications enable text, neuroimaging, photos, and videos to be shared securely among the stroke team. Our aim was to investigate whether use of a smartphone application would shorten the duration from admission to reperfusion therapy in patients with hyper-acute ischemic stroke.

Methods Enrolled were acute ischemic stroke patients who underwent reperfusion therapy (intravenous tissue plasminogen activator (IV t-PA) and mechanical thrombectomy (MT)) at our hospital between October 2012 and September 2018. We divided the patients into two groups based on the date of availability on smartphones of communication-type medical application: (1) Control group, conventional communication prior to September 2015, and (2) App group, communication via the smartphone app from October 2015 onwards. We compared door-to-image time (DIT), image-to-needle time (INT), door-to-needle time (DNT) for thrombolysis, and DIT, image-to-puncture time (IPT), and door-to-puncture time (DTP) for thrombectomy between the groups. Results We retrospectively enrolled 139 patients (68\% male; median age, 69 years; median NIHSS score, 7) who were assigned into the App group $(n=86)$ and Control group $(n=53)$. Of the overall patients, 109 underwent IV t-PA (IV t-PA alone, 79 patients), and 63 underwent MT (MT alone, 30 patients), and 33 patients underwent combined IV t-PA and MT. There was no significant difference in DIT between the App and Control groups ( $23 \mathrm{~min}$ vs. $22 \mathrm{~min}, p=0.493$ ). DNT, DPT, INT, and IPT were significantly shorter in the App group than in the Control group (DNT, 62 min for the App group vs. 72 min for Control group, $p$ $=0.038$; INT, 42 vs. $48 \mathrm{~min}, p=0.009$; DPT, 106 vs. $129 \mathrm{~min}, p=0.046$; IPT, 89 vs. $117 \mathrm{~min}, p=0.004$ ).

Conclusion The present findings indicate that communication-type medical smartphone apps have potential for shortening the time elapsed between admission and reperfusion therapy, especially INT and IPT.
\end{abstract}

Keywords Smartphone application $\cdot$ Reperfusion therapy

\section{Introduction}

The effectiveness of administration of intravenous tissue plasminogen activator (IV-tPA) and mechanical thrombectomy

Kenichiro Sakai

k.sakai.0127@gmail.com

1 Department of Neurology, The JIKEI University School of Medicine, Tokyo, Japan

2 Department of Neurosurgery, The JIKEI University School of Medicine, Tokyo, Japan

3 Department of Innovation for Medical Information Technology, The JIKEI University School of Medicine, Tokyo, Japan
(MT) has been proven for treatment of patients with acute ischemic stroke [1-5]. A meta-analysis found that the benefits are highly time dependent: earlier IV-tPA and MT are associated with reduced disability, mortality, and risk of complications such as symptomatic intracranial hemorrhage [6-10]. Therefore, after the decision has been made to administer this therapy, the stroke team must initiate the treatment as soon as possible.

At hospitals where reperfusion therapy is performed, it is extremely important to establish a stroke care system to shorten the time taken at each of the steps between onset and reperfusion, i.e., from admission to imaging, imaging to puncture, and puncture to recanalization. The Target: Stroke initiative [7] developed a stroke algorithm system designed to reduce the time taken until treatment that codes single-call activation systems, stroke tools, rapid brain imaging, and laboratory testing. 
Recently, social networks using smart device such as smartphones and tablet terminal have become widespread and indispensable tool for patients and neurologists [11, 12]. Furthermore, it has been suggested that this digitization process may be accelerated in the stroke care after a global emergency represented by the COVID-19 pandemic [13]. Already, several stroke centers have trialed a medical information and communication technology (ICT) smartphone applications $[14,15]$ with the aim of shortening IV-tPA and MT times from onset. These applications perform task management and time tracking for reperfusion therapy, and present this information visually for sharing among the stroke team.

In the present study, we used a communication-type medical ICT smartphone application to build a seamless system for information sharing within the stroke team, similar to a social networking service. Within the application, the team can share texts, neuroimaging, photos, and videos with high security. This information be referred to independently at any time by each member of the specialized stroke team, whether they are physically at the hospital or not, for decision making regarding reperfusion therapy. Some previous studies have reported a reduction in reperfusion therapy time by using a smartphone application as a communication tool, but the applications did not incorporate a time tracker. The aim of the present study was to evaluate the ability of a communication-type medical application to reduce the time from hospitalization to reperfusion therapy, and thus improve patients' functional outcomes.

\section{Methods}

The authors declare that all supporting data are available within the article.

\section{Study design}

This retrospective study included consecutive ischemic stroke patients with acute ischemic stroke who underwent thrombolysis and/or mechanical thrombectomy between October 2012 and September 2018. Patients' clinical information, including cardiovascular risk factors (hypertension, diabetes mellitus, dyslipidemia, smoking status) and past history of atrial fibrillation, was obtained from the Jikei University School of Medicine Stroke Registry (Jikei Stroke Registry), a prospective database of patients with acute stroke admitted to Jikei University Hospital, a tertiary medical center located in the center of the Tokyo metropolitan area. Stroke severity on admission was assessed using the National Institutes of Health Stroke Scale (NIHSS) score [16]. Using the criteria of the Trial of ORG 10172 in Acute Stroke Treatment (TOAST) [17], stroke subtype was categorized into five groups: smallvessel occlusion, large-artery atherosclerosis, cardioembolism, other determined etiology, and undetermined.
Our stroke center has communication-type medical application JOIN (Allm Inc, Tokyo, Japan) to sharing of neuroimages and teleconsultation. This smartphone application has a different purpose than neuroimaging and teleconsulting. This app allows you to post short message services (SMS) and DICOM images to authorized groups, reducing the effort and hurdles required for sharing information within the Stroke Team. This allows Stroke Team members to make quick decisions even when they are in remote locations or organizations. In addition, the user interface similar to application of social networking services allows intuitive sharing of medical information such as diagnostic images (CT, MRI, and ultrasonography) with the stroke team [18]. JOIN is GDPR (General Data Protection Regulation) and HIPPA (Health Insurance Portability and Accountability Act) compliant and meets privacy standards in many countries.

This study conformed to the ethical principles established in the Declaration of Helsinki, and the Institutional Review Board of Jikei University School of Medicine, Japan, approved the study protocol (No. 8813). The board waived the need for patient consent.

\section{Statistical analysis}

We divided the patients into two groups based on the date of availability on smartphones of communication-type medical application: (1) Control group, conventional communication prior to September 2015, and (2) App group, communication via the smartphone application from October 2015 onwards. We compared door-to-image time (DIT), image-to-needle time (INT), door-to-needle time (DNT) for thrombolysis, and DIT, image-to-puncture time (IPT), and door-topuncture time (DTP) for thrombectomy between the groups. Continuous variables were summarized using medians (interquartile range [IQR]) and categorical variables were expressed as counts $(\%)$. We compared the clinical backgrounds, elapsed time (DIT, INT, DNT, IPT, DPT), efficacy, and safety outcomes (recanalization rate at $24 \mathrm{~h}$ after reperfusion therapy, symptomatic intracranial hemorrhage at $24 \mathrm{~h}$, neurologic deterioration at $24 \mathrm{~h}$, modified Rankin scale [mRS] at discharge and 90 days, mortality at 90 days) between the two groups using the chi-square test or Mann-Whitney $U$ test, as appropriate. Symptomatic intracranial hemorrhage was defined according to the European Cooperative Acute Stroke Study III criteria [2] as the presence of extravascular blood in the cranium that was associated with an increase in the NIHSS score of $\geq 4$ points or with death, and judged to be the predominant cause of neurologic deterioration. Recanalization was defined as modified MORI grade $>2$ on MR angiography [19]. Neurologic deterioration was defined as an increase in NIHSS score of $\geq 4$ points within $24 \mathrm{~h}$ after stroke that was not attributed to intracranial hemorrhage or malignant cerebral 
edema. A two-tailed $P<0.05$ was considered statistically significant. All statistical analyses were performed using SPSS for Windows version 25.0 (SPSS, Chicago, IL).

\section{Results}

\section{Clinical background}

We retrospectively enrolled 139 consecutive patients $(68 \%$ male; median age, 69 years; median NIHSS score, 7). Table 1 lists patients' clinical characteristics. Of the 139 patients, 86 were assigned into the App group and 53 were assigned into the Control group. Of all patients, 109 underwent IV t-PA, of which 76 underwent IV t-PA alone; 63 underwent MT, of which 30 underwent MT alone; and 33 patients underwent combined IV t-PA and MT.

\section{IV-tPA after admission}

Sixty-four patients in the App group and 45 patients in the Control group were treated with IV-tPA. There was no significant difference in DIT between the App and Control groups (23 min vs. 22 min, $p=0.493$ ). In contrast, INT and DNT were significantly shorter in the App group than the Control group (INT, 42 min for the App group vs. 48 min for the
Control group, $p=0.006$; DNT, 62 vs. $72 \mathrm{~min}, p=0.038$, Fig. 1).

\section{Mechanical thrombectomy after admission}

Forty-two patients in the App group and 21 patients in the Control group underwent mechanical thrombectomy. There was no significant difference in terms of DIT between the two groups ( 22 vs. $23 \mathrm{~min}, p=0.493$ ). However, IPT and DPT were significantly shorter in the App group than in the Control group (IPT, 75 vs. $94 \mathrm{~min}, p=0.002$; DPT, 100 vs. 129 min, $p=0.022$; Fig. 2).

\section{Efficacy and safety outcomes}

Table 2 shows the efficacy and safety outcomes for the two groups. There was no statistically significant difference between the App and Control groups from onset to $24 \mathrm{~h}$ after onset in terms of recanalization rate, symptomatic intracranial hemorrhage, or neurologic deterioration (recanalization, $66 \%$ vs. $72 \%, p=0.53$; symptomatic intracranial hemorrhage, $1 \%$ vs. $4 \%, p=0.264$; neurologic deterioration, $9 \%$ vs. $15 \%, p=$ 0.216). In terms of outcome, there was no difference in $\mathrm{mRS}$ at discharge and 90 days between the App and Control groups (mRS at discharge, 2 vs. $2, p=0.215$; $\mathrm{mRS}$ at 90 days, 1 vs. 2 , $p=0.554)$. Of note, mortality at 90 days was lower in the App

Table 1 Patient characteristics

\begin{tabular}{|c|c|c|c|}
\hline & App group $(n=86)$ & Control group $(n=53)$ & $p$ \\
\hline Age (years), median (IQR) & $66(54-79)$ & $71(64-78)$ & 0.184 \\
\hline Male, $n, \%$ & $57(66)$ & $37(70)$ & 0.405 \\
\hline \multicolumn{4}{|l|}{ Past history } \\
\hline Hypertention, $n, \%$ & $52(61)$ & $38(72)$ & 0.122 \\
\hline Hyperlipidemia, $n, \%$ & $28(33)$ & $26(49)$ & 0.04 \\
\hline Diabetes melllitus, $n, \%$ & $20(23)$ & $12(23)$ & 0.553 \\
\hline Atrial fibrillation, $n, \%$ & $19(22)$ & $14(26)$ & 0.351 \\
\hline Prestroke mRS, median (IQR) & $0(0-1)$ & $0(0-0)$ & 0.062 \\
\hline NIHSS score on admission, median (IQR) & $6(3-15)$ & $9(5-18)$ & 0.027 \\
\hline TOAST classification, $n, \%$ & & & $<0.01$ \\
\hline Large-artery atherosclerosis, $\mathrm{n}, \%$ & $8(9)$ & $15(28)$ & \\
\hline Small-vessel occlusion, $n, \%$ & $4(5)$ & $2(4)$ & \\
\hline Cardioembolism, $n, \%$ & $33(38)$ & $25(47)$ & \\
\hline Other determined ethiology, $n, \%$ & $3(4)$ & $5(10)$ & \\
\hline Undetermined, $n, \%$ & $28(33)$ & $6(11)$ & \\
\hline Major artery occlusion, $n, \%$ & $62(72)$ & $43(81)$ & 0.692 \\
\hline Treatment & & & 0.332 \\
\hline $\mathrm{tPA}, n, \%$ & $44(51)$ & $32(60)$ & \\
\hline Mechanical thrombectomy, $n, \%$ & $22(26)$ & $8(15)$ & \\
\hline tPA+mechanical thrombectomy, $n, \%$ & $20(23)$ & $13(25)$ & \\
\hline
\end{tabular}

$m R S$ modified Rankin scale, NIHSS national institute health stroke scale, TOAST Trial of ORG 10172 in Acute Stroke Treatment 
Fig. 1 Time from admission to initiation of IV-tPA. INT and DNT were significantly shorter in the App group than the Control group. DIT door to image time, INT image to needle time, DNT door to needle time

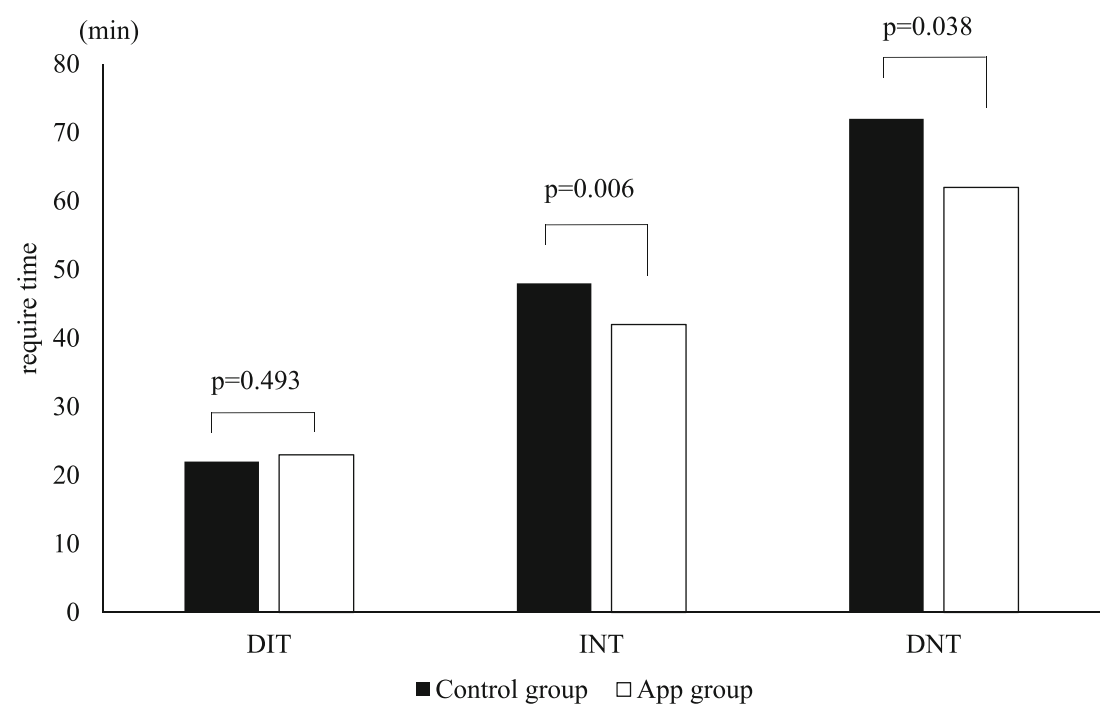

group than the Control group ( $2 \%$ vs. $13 \%, p=0.014)$. There was no difference between the groups in terms of mRS at 90 days in patients who underwent tPA alone. However, the percentage of good outcome at 90 days showed a tendency to be higher in patients who underwent MT alone, IV t-PA with MT, or MT with or without IV t-PA (supplementary 1).

\section{Discussion}

The results of the present study showed shorter DNT and DPT values for patients in the App group. In particular, treatment was most rapid for patients in this group who underwent MT. In addition, there were no difference DPT between the App group and Control group, but INT and IPT was shorter in the App group. The mRS at discharge and 90 days was similar between the groups, although mortality at 90 days was lower in the App group.

Our study found that use of the smartphone app reduced the DNT and DPT values. Specifically, shortening INT and IPT contributed to shortening DNT and DPT. A previous study has reported that use of a medical acute care coordination app decreased mean DNT by $40 \mathrm{~min}[20,21]$; however, they did not investigate the cause of shortened DNT in detail. We consider that DNT and DPT were shorter with use of the smartphone app for the following reasons. Cooperation within the professional stroke treatment team is very important in reperfusion therapy. After rapid medical examination and neuroimaging have been performed, the stroke patient is transported to the ward, the operating room/angiography room is prepared, and the cerebrovascular specialist, anesthesiologist, and ICU/SCU physicians are contacted. Arranging each of these steps conventionally by telephone is time intensive; in
Fig. 2 Time from admission to initiation of mechanical thrombectomy. IPT and DRT were significantly shorter in the App group than the Control group. DIT door to image time, INT image to needle time, DNT door to needle time

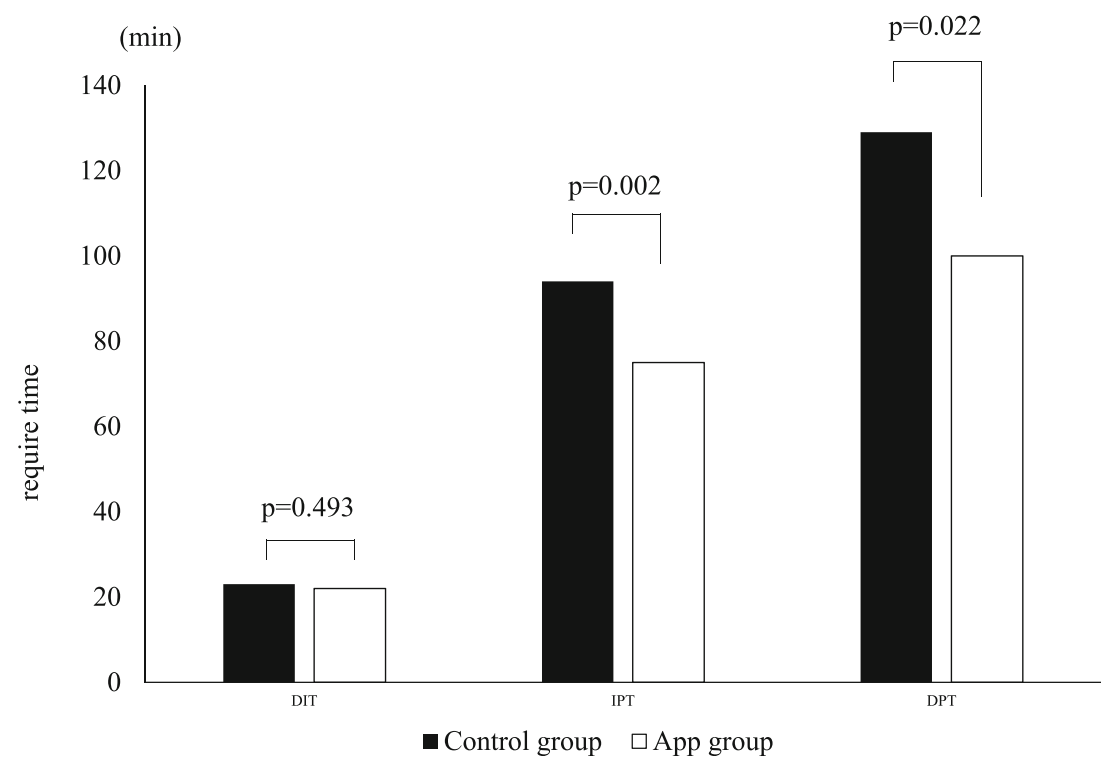


Table 2 Efficacy and safety outcomes in the Smartphone app and Control groups

\begin{tabular}{llll}
\hline & App group $(n=86)$ & Control group $(n=53)$ & $p$ \\
\hline Recanalization at $24 \mathrm{~h}^{*}, n, \%$ & $57(66)$ & $38(72)$ & 0.53 \\
Symptomatic intracranial hemorrhage at $24 \mathrm{~h}^{* *}, n, \%$ & $1(1)$ & $2(4)$ & 0.264 \\
Neurologic deterioration at $24 \mathrm{~h}^{* * *}, n, \%$ & $8(9)$ & $8(15)$ & 0.216 \\
discharge $\mathrm{mRS}$ & $2(1-4)$ & $2(1-4)$ & 0.215 \\
90 days mRS & $1(0-4)$ & $2(0-4)$ & 0.554 \\
90 days mRS $<2$ & $55(65)$ & $32(63)$ & 0.480 \\
90 days mortality, $n, \%$ & $2(2)$ & $7(13)$ & 0.014 \\
\hline
\end{tabular}

*Recanalization was defined as modified MORI grade $>2$ with MR angiography

**Symptomatic intracranial hemorrhage was defined according to European Cooperative Acute Stroke Study III criteria as the presence of extravascular blood in the cranium that was associated with an increase in the NIHSS score of 4 points or more or death and was judged to be the predominant cause of neurologic deterioration

***Neurologic deterioration was defined as an increase in the NIHSS score of 4 or more points within $24 \mathrm{~h}$ after stroke that was not attributed to intracranial hemorrhage or malignant cerebral edema

$m R S$ modified Rankin Scale comparison, communication via a smartphone app using a server-based network can instantly share patient medical information among the stroke team, including text and neuroimaging, in one simple action. Because it is available immediately after sharing, medical information can be evaluated via common chat room interfaces, thereby facilitating real-time discussions by the team. Task manager-type and time tracker-type apps cannot perform the function of communication among the stroke team. We consider that using the smartphone app saved process and communication time in coordinating reperfusion therapy, which requires collaboration of an interdisciplinary team.

Use of the smartphone app shortened DNT and DPT but was not related to favorable outcome at 3 months, for the reason that our cohort contained many patients with non-severe stroke. The favorable outcome rate was very high in both of the present groups compared with those reported previously [9, 22]. Previous studies have reported that in-hospital medical apps $[15,20]$, telestroke evaluation [23], and treatment by a mobile stroke unit $[23,24]$ reduced DNT, but to the best of our knowledge, none studied the relationship between favorable outcome and improvements in the stroke care system.

There are some limitations of the present study. We speculate that our results may have been affected by its small sample size, single-center design, retrospective cohort, and efforts to reduce time for IV t-PA and MT for code stroke system. Further large-scale trials are required to confirm the effect of the smartphone app on reducing time to initiation of reperfusion therapy.

\section{Conclusion}

Use of a communication-type medical smartphone app reduced the time from admission to initiation of reperfusion therapy compared with the conventional communication method. The time saving effect was higher for MT than for IV t-PA.

Supplementary Information The online version contains supplementary material available at https://doi.org/10.1007/s10072-021-05132-2.

\section{Declarations}

Ethical approval This article does not contain animals performed by any of the authors. All procedures performed in studies involving human participants were in accordance with the ethical standards of the institutional research ethics committee and with the 1964 Helsinki declaration and its later amendments or comparable ethical standards.

Informed consent Informed consent was obtained from all individual participants included in the study.

Conflict of interest The authors declare that they have no conflict of interest.

\section{References}

1. National Institute of Neurological Disorders and Stroke rt-PA Stroke Study Group (1995) Tissue plasminogen activator for acute ischemic stroke. N Engl J Med 333:1581-1587

2. Hacke W, Kaste M, Bluhmki E, Brozman M, Dávalos A, Guidetti D et al (2008) Thrombolysis with alteplase 3 to 4.5 hours after acute ischemic stroke. N Engl J Med 359:1317-1329

3. Berkhemer OA, Fransen PSS, Beumer D, van den Berg LA, Lingsma HF, Yoo AJ et al (2015) A randomized trial of intraarterial treatment for acute ischemic stroke. N Engl J Med 372:11-20

4. Goyal M, Demchuk AM, Menon BK, Eesa M, Rempel JL, Thornton J et al (2015) Randomized assessment of rapid endovascular treatment of ischemic stroke. N Engl J Med 372: 1019-1030 
5. Campbell BCV, Mitchell PJ, Kleinig TJ, Dewey HM, Churilov L, Yassi $\mathrm{N}$ et al (2015) Endovascular therapy for ischemic stroke with perfusion-imaging selection. N Engl J Med 372:1009-1018

6. Hacke W, Donnan G, Fieschi C, Kaste M, Kummer von R, Broderick JP et al (2004) Association of outcome with early stroke treatment: pooled analysis of ATLANTIS, ECASS, and NINDS rtPA stroke trials. Lancet 363:768-774

7. Fonarow GC, Smith EE, Saver JL, Reeves MJ, Hernandez AF, Peterson ED et al (2011) Improving door-to-needle times in acute ischemic stroke: the design and rationale for the American Heart Association/American Stroke Association's Target: Stroke initiative. Stroke. 42:2983-2989

8. Saver JL, Fonarow GC, Smith EE, Reeves MJ, Grau-Sepulveda MV, Pan W, Olson DWM, Hernandez AF, Peterson ED, Schwamm LH (2013) Time to treatment with intravenous tissue plasminogen activator and outcome from acute ischemic stroke. JAMA 309:2480-2488

9. Badhiwala JH, Nassiri F, Alhazzani W, Selim MH, Farrokhyar F, Spears J, Kulkarni AV, Singh S, Alqahtani A, Rochwerg B, Alshahrani M, Murty NK, Alhazzani A, Yarascavitch B, Reddy K, Zaidat OO, Almenawer SA (2015) Endovascular thrombectomy for acute ischemic stroke: a meta-analysis. JAMA 314:1832-1843

10. Lin Y, Schulze V, Brockmeyer M, Parco C, Karathanos A, Heinen Y, Gliem M, Hartung HP, Antoch G, Jander S, Turowski B, Perings S, Kelm M, Wolff G (2019) Endovascular thrombectomy as a means to improve survival in acute ischemic stroke: a meta-analysis. JAMA Neurol 76:850-854

11. Lavorgna L, Brigo F, Abbadessa G, Bucello S, Clerico M, Cocco E, Iodice R, Lanzillo R, Leocani L, Lerario A, Moccia M, Padovani A, Prosperini L, Repice A, Stromillo M, Trojsi F, Mancardi G, Tedeschi G, Bonavita S (2020) The use of social media and digital devices among Italian neurologists. Front Neurol 11:583

12. Moccia M, Brigo F, Tedeschi G, Bonavita S, Lavorgna L (2018) Neurology and the Internet: a review. Neurol Sci 39(6):981-987

13. Iodice F, Romoli M, Giometto B, Clerico M, Tedeschi G, Bonavita S, et al(2021) Stroke and digital technology: a wake-up call from COVID-19 pandemic. Neurol Sci 42:805-809

14. Dickson RL, Sumathipala D, Reeves J (2016) Stop Stroke@ acute care coordination medical application: a brief report on postimplementation performance at a primary stroke center. J Stroke Cerebrovasc Dis 25:1275-1279

15. Matsumoto S, Koyama H, Nakahara I, Ishii A, Hatano T, Ohta T et al (2019) A visual task management application for acute ischemic stroke care. Front Neurol. Frontiers 10:1118

16. Adams HP, Davis PH, Leira EC, Chang KC, Bendixen BH, Clarke WR et al (1999) Baseline NIH Stroke Scale score strongly predicts outcome after stroke: a report of the Trial of Org 10172 in Acute Stroke Treatment (TOAST). Neurology 53:126-131

17. Adams HP, Bendixen BH, Kappelle LJ, Biller J, Love BB, Gordon DL et al (1993) Classification of subtype of acute ischemic stroke.
Definitions for use in a multicenter clinical trial. TOAST. Trial of Org 10172 in Acute Stroke Treatment. Stroke 24:35-41

18. Sakai K, Komatsu T, Iguchi Y, Takao H, Ishibashi T, Murayama Y (2020) Reliability of smartphone for diffusion-weighted imagingalberta stroke program early computed tomography scores in acute ischemic stroke patients: diagnostic test accuracy study. J Med Internet Res 22:e15893

19. Mori E, Minematsu K, Nakagawara J, Yamaguchi T, Sasaki M, Hirano T, Japan Alteplase Clinical Trial II Group (2010) Effects of $0.6 \mathrm{mg} / \mathrm{kg}$ intravenous alteplase on vascular and clinical outcomes in middle cerebral artery occlusion: Japan Alteplase Clinical Trial II (J-ACT II). Stroke 41:461-465

20. Andrew BY, Stack CM, Yang JP, Dodds JA (2017) mStroke: "Mobile stroke"-improving acute stroke care with smartphone technology. J Stroke Cerebrovasc Dis Elsevier Inc 26:1449-1456

21. Dickson R, Nedelcut A, McPeek M (2017) Stop Stroke: a brief report on door-to-needle times and performance after implementing an acute care coordination medical application and implications to emergency medical services. Prehospital and Disaster Medicine. 32:343-347.

22. Goyal M, Menon BK, van Zwam WH, Dippel DWJ, Mitchell PJ, Demchuk AM, Dávalos A, Majoie CBLM, van der Lugt A, de Miquel MA, Donnan GA, Roos YBWEM, Bonafe A, Jahan R, Diener HC, van den Berg LA, Levy EI, Berkhemer OA, Pereira VM, Rempel J, Millán M, Davis SM, Roy D, Thornton J, Román LS, Ribó M, Beumer D, Stouch B, Brown S, Campbell BCV, van Oostenbrugge RJ, Saver JL, Hill MD, Jovin TG (2016) Endovascular thrombectomy after large-vessel ischaemic stroke: a meta-analysis of individual patient data from five randomised trials. Lancet 387:1723-1731

23. Taqui A, Cerejo R, Itrat A, Briggs FBS, Reimer AP, Winners S, Organek N, Buletko AB, Sheikhi L, Cho SM, Buttrick M, Donohue MM, Khawaja Z, Wisco D, Frontera JA, Russman AN, Hustey FM, Kralovic DM, Rasmussen P, Uchino K, Hussain MS, Cleveland Pre-Hospital Acute Stroke Treatment (PHAST) Group (2017) Reduction in time to treatment in prehospital telemedicine evaluation and thrombolysis. Neurology 88:1305-1312

24. Kunz A, Ebinger M, Geisler F, Rozanski M, Waldschmidt C, Weber JE, Wendt M, Winter B, Zieschang K, Fiebach JB, Villringer K, Erdur H, Scheitz JF, Tütüncü S, Bollweg K, Grittner U, Kaczmarek S, Endres M, Nolte CH, Audebert HJ (2016) Functional outcomes of pre-hospital thrombolysis in a mobile stroke treatment unit compared with conventional care: an observational registry study. Lancet Neurol 15:1035-1043

Publisher's note Springer Nature remains neutral with regard to jurisdictional claims in published maps and institutional affiliations. 